\title{
Open-ended Questions and Mixed-mode Survey? A Comparison between Telephone Survey and Online Survey
}

\author{
Dr. Mohammad Hatim Abuljadail \\ Associate Professor \\ Department of Marketing Communication \\ King Abdulaziz University \\ Kingdom of Saudi Arabia
}

\begin{abstract}
The emergence of Internet has help surveyors create online survey methodology which had advantages and disadvantages. One of the online survey disadvantages is its difficulty in applying it on a random sample. Therefore, many researchers complement the disadvantages in any survey mode by using another mode. Although the use of mixed-mode became the new norm, previous studies have raised questions about the measurement of the questions and its reliability in measuring the same answers in two different modes. This study investigates one particular type of survey questions (open-ended) and compare the item-response rate between two groups of participants that responded to the survey through two different modes (telephone and online). The findings of the study show significant difference between the two groups in the item-response rate of open-ended questions. Online participants answer more open-ended questions than telephone participants. The study offers suggestions for future research and discusses the limitations in this study.
\end{abstract}

Keywords: Online, Telephone, Mixed-mode, Survey, Open-ended, Item Response.

\section{Introduction}

Survey as a methodology has been through many changes and shifts in the mode used to collect data (Dillman, Smyth \& Christian, 2009). Prior to the 1970s, face-to-face interviewing was the mode of collecting data for studies that sought high response rates and generalizability (Dillman et al., 2009). However, since the 1970s, the cost of face-to-face interviewing has increased, which led researchers to look for other affordable modes to collect data, such as telephone survey, paper-and-pencil mail survey, audio computer-assisted self-interviewing and other alternatives that are computer assisted surveys (Dillman et al., 2009). Telephone survey studies that investigated samples generated by random digit dialing, gained popularity due to its quality and ability to reach many respondents. In addition, the ease of contacting respondents at random and low cost increased the appeal of telephone surveys. Through time and growth of modern technology, other ways of collecting information were developed such as internet surveys.

Many factors go into the use of surveys in research such as the type of survey conducted as well as the kinds of questions asked. If the interview was self-paced or facilitated by a trained interviewer and how the questions were posed, all make a difference in the reliability of the responses. For the purposes of this paper we will discuss these differences in terms of online and telephone surveys and how they relate to responses and nonresponses. In addition, we will compare how internet and telephone respondents answer survey opened-ended questions. The purpose of investigating this research question is that a particular question could

* This article was submitted in March 2021, and accepted for publishing in April 2021.

(c) Arab Administrative Development Organization- League of Arab States, 2023, pp 227-236،

DOI: 10.21608/aja.2021.63746.1047 
be answered differently if asked through two different modes (Dillman et al., 2009). The popularity of mixedmode has raised many questions about the reliability of the survey questions. Therefore, this study will focus on a particular type of survey question (open ended) and will investigate if these questions will be answered differently from one mode to another (telephone and online).

\section{Literature Review}

\section{Mixed-mode Survey}

The use of mixed-mode survey has become popular and some scholars consider it as the norm of conducting a survey (Biemer \& Lyberg, 2003). Technological development has helped surveyors to integrate mixed-mode methodology in their research; technologies not only helped researchers in the data collection phase, but in many ways such as the design of the survey and the analysis of the data collected through the mixed-mode (Dillman et al., 2009). In addition to the technological development, surveyors have been eagerly trying to minimize survey errors such as coverage, sampling and non-response error (Dillman et al., 2009). For example, researchers can reduce coverage error when one mode cannot cover the intended population, thus, the addition of another mode could improve coverage and help surveyors reach those who were not reached in the first mode (Dillman et al., 2009). In this study, members of the population provided different ways of contact; some provided emails while others provided phone numbers. Therefore, a mixed mode (telephone and online) is beneficial to improve coverage.

One mode could cover the population of interest, but still many individuals might not respond. People have different preferences in how they like to be surveyed; some individuals like to participate online whole others prefer to participate on the phone. Therefore, mixed-mode can reduce non-response error by providing another mode to those who did not respond to the first mode (Dillman et al., 2009). Mixed-mode surveys can also reduce measurement errors - especially when asking sensitive questions such as sexuality or the use of drugs or alcohol. Previous studies have shown that respondents prefer to answer sensitive questions online than face-to-face or over the phone. Asking sensitive questions over the phone or face to face could lead to answers that are socially desirable instead of the real answers of the particular respondents (Dillman et al., 2009). The use of mixed-mode survey can also reduce cost and provide faster results (Dillman et al., 2009). Researchers could start with the more economic mode and then try to reach the more difficult participants through the more expensive mode. Further, studies that have time limitations could choose the mode that leads to faster results; they can also use both modes simultaneously (Dillman et al., 2009).

The data collected are a contribution of the combination of the mixed-mode. The decision of choosing the modes should be made carefully by the research team and that decision should consider total survey error, target population, budget and research questions (Dillman et al., 2009).

Previous studies examined how using different survey modes could lead to different answers. A simple question such as "what is your marital status?" was answered differently over the phone than online; when it was asked over the phone, respondents answered by either "married" or "single" and the interviewer checked the answer of the five options they have. On the other hand, respondents who answered the online survey were able to see the five options and some of them chose divorced, widowed or separated (Dillman et al., 2009). This example shows how mixed-mode could produce different responses from one mode to another. This example also shows another important issue; it illustrates the differences between open-ended and closed-ended questions. Telephone respondents answered the question as an open-ended question, which limited the answer to "married" or "single" while online respondents answered the questions as a closed-ended questions, which prompted respondents to give more detailed answer such as "widowed", 
"divorced" or "separated" (Dillman et al., 2009). Beside the communication issue, sometimes researchers purposely simplify the questions asked to telephone respondents in order to make it easier; answering longer and complicated questions over the phone is more difficult and distracting than answering it face-toface or online. Therefore, some surveyors shorten some questions or reduce the number of categories in order to make the questions easier to understand and follow (Dillman et al., 2009).

Further, there are ways that help survey designers to force respondents to answer questions. For example, surveyors can implement a function that forces online respondents to choose an answer in order to proceed to the next question. Studies have shown that respondents are more likely to answer the questions when a force function is implemented (Dillman et al., 2009).

\section{Telephone Surveys}

Telephone surveys have long been one of the most commonly used modes of conducting surveys. Due to the ease of contacting randomly selected participants and being relatively inexpensive, telephone surveying is a largely utilized method for collecting information. In the 1990s, roughly $94 \%$ of all American households had a landline telephone (Czaja \& Blair, 1996). Furthermore, the ability to reach a truly random respondent group and one that most accurately reflects the demographics relevant to the researchers' needs, have made telephone surveys one major preferred method.

In order to reach a completely random sample, telephone surveyors have several options for obtaining telephone numbers. One very common method is random digit dialing (RDD) where numbers are selected at random from a telephone book, randomly dialed by changing only the one to four digits of a known telephone number or obtaining them from a membership directory or other group listing - telephone numbers can even be purchased at a relatively low cost. Due to the ease of reaching valid telephone numbers, interviewers can usually make contact with a significant number of respondents at any time of the day (Czaja \& Blair, 1996).

Telephone surveys have many advantages over mail surveys or other non-telephone surveys for several reasons. The mere presence of an interviewer can entice many who answer their phone to continue the conversation. There is also likely to be more accurate and unbiased answers given because respondents cannot read ahead or know in advance what question will be asked. For many surveys, a "yes" response yields another series of questions that respondents may not want to answer. On a paper survey, respondents might just enter "no" in order to avoid the following questions. Not having the ability to scan ahead and pre-meditate answers helps to make telephone interviews quite accurate and sincere. There is also the advantage of being able to reach a larger group of people as respondents may not have the ability to read, the ability to access a computer or navigate the internet or will not simply set the survey aside and forget about it. With the spontaneity of conversation, interviewers can prompt respondents for answers and since the surveys are generally fairly short, respondents are more likely to finish the conversation (Czaja \& Blair, 1996).

While there are many advantages to telephone surveys over other methods, there are weaknesses as well. Interviewers should be hired from the outside, adding cost and time to the research. Interviewers must also be trained in how to conduct surveys appropriately. For example, each question must be asked exactly as written, in the same order and interviewers cannot display any form of bias in their tone of voice or in how the questions are worded. It is important that surveyors be trained which adds to the time and cost of the study (Czaja \& Blair, 1996). Furthermore, questions must remain short so that respondents won't mind taking the time to do them. Since it is a telephone call, respondents have to feel like it is not taking too much of their time. Questions must be direct to avoid respondents either answering quickly without really 
thinking about their answer or simply refusing to participate in the first place. It is also easy for respondents to forget parts of the question or answer selections which could yield inaccurate results. There is also the concern that if the respondent cannot think of an answer right away there is awkward silence-potentially prompting quick and biased answers. Often times when the question is of a personal nature, some people may be too self-conscious to respond honestly. Studies have shown that respondents in oral interviews will fix their answers in order to present themselves more favorably (Fowler, Roman \& Di, 1998). In self-administered questionnaires there is the security of anonymity whereby respondents don't have to worry as much about their self-image or what others will think of their responses.

In addition, in more recent times with the increased popularity of wireless telephone service, many have disconnected their landline telephone service altogether. In 2007 nearly 16\% of Americans used only their wireless telephone (Dillman et al., 2009). Consequently fewer people have their phone number listed in a telephone directory, more people are screening their phone calls using caller ID or letting calls go to voicemail and it is very difficult to track the demographics of the respondent group when numbers are wireless as we cannot know who they are or where they reside based only on their telephone number. Lastly, there is the disadvantage of not being able to record non-respondents or non-contacts. Because often times telephone surveys are conducted using RDD, interviewers may not know who they are dialing and if it is actually a number to a household or even if it will be a working number. This can make non-responses difficult to organize and record.

\section{Internet Surveys}

With modern technology and the increased popularity of the internet, more and more surveys can be done online. Especially now that large portions of society are relying on their computers and even smartphones for telephone and email access, online surveys are a viable means of collecting data. One way that online surveys reach respondents is through self-selected panels where people volunteer to join and take part in the survey. Other organizations can have relationships with members that inform people prior to joining that they could be asked to participate in research surveys thus allowing researchers to reach out to those people when respondents are sought. Internet surveys differ from telephone surveys in that random sample surveys cannot be so readily sent. Email addresses are not as predictable nor are they as random, as telephone numbers (Dillman et al., 2009).

There are advantages of online surveys in that they can be taken at a slower pace which could increase the likelihood of someone actually completing the survey. Without the pressure of an interviewer waiting for an answer, one can take their time and in addition, can be more thoughtful about their answers. This will yield more honest answers and more reliable results. This also alleviates the problem of biased answers. Unlike in face-to-face interviews, online surveys allow respondents the privacy of knowing that they can answer sensitive questions openly without risk of being embarrassed or feeling ashamed if their answer is less than socially desirable (Chang \& Krosnick, 2009).

There are many disadvantages with internet surveys however. While internet is widely used, studies have shown that in 2007 only 67\% of American households had internet at their home (Dillman et al., 2009). Though more than that has knowledge of how to use the internet, access to surveys from home is limited when compared to telephone surveys. Furthermore, use of the internet can lead to samples that do not represent certain groups. For example, senior citizens might be less knowledgeable about navigating the web versus college students and younger professionals. That many surveys are sent using email lists from organizations; those respondents are likely to have similar interests and backgrounds leading to inaccurate overall results. Even though a study may report results from thousands of respondents, those respondents may not necessarily be representative of the population. 


\section{Nonresponse and Item-nonresponse Errors}

Nonresponse happens when the sample members do not respond to the survey or some of the survey (Groves, 2004). Nonresponse rate is increasing due to the development of technologies; individuals now can block phone calls or let the voice mail machine answer the calls for them. There are many ways for potential respondents to avoid participating. Therefore, nonresponse rate and nonresponse error have become an emerging topic of interest for social scientists around the world (Dillman, Eltinge, Groves \& Little, 2002). The purpose of using survey as a method is usually to collect data that can represent the population, and its results can be generalizable to the population (Hoonakker \& Carayon, 2009). High response rate is highly desirable and reflects on the quality of the survey and the responses (Hox \& De Leeuw, 1994).

One of the factors that affects response quality is the number of questions answered (Hoonakker \& Carayon, 2009). The more answered questions the better the quality of the survey and results. The amount of answers matters even in open-ended questions; longer answers in open-ended questions are an indicator of a good quality survey (Schaefer \& Dillman, 1998).

\section{Open-ended Questions in a Survey}

The design of every research question depends on how the construct will be measured (Dillman et al., 2009). For example, some survey questions are measured better when they are asked in an open-ended format while others are better asked in a closed-ended format. For the purpose of this study, the paper will focus on open-ended survey questions and its categories. There are three different main types of survey open-ended questions: open-ended questions for numerical answers, open-ended questions for a list of items, and open-ended questions foe descriptive answers (Dillman et al., 2009). Each of these open-ended questions types give respondents the flexibility to answer the questions with freedom; however, the answers could still be influenced by the wording of the question, the way they are asked, and the visual design of the question (Dillman et al., 2009). In addition, the flexibility that is given to the respondent to answer the open-ended questions could affect the measurement of the constructs; each type of the three open-ended questions types requires a different answer format as well as a different measurement (Dillman et al., 2009). For example, it is undesirable to provide descriptive answers in open-ended questions that require numerical answers.

In open-ended questions that require a numerical answer, respondents are asked to enter an amount or a particular number in a box. Researchers are encouraged to carefully design the question in order to minimize undesirable answers that could cause problems during data entry and cleaning (Dillman et al., 2009). There are several strategies that help surveyors to design numerical open-ended questions that lead to desirable responses, such as asking specifically for the desired unit to answer the question, sizing the answer box according to the desired answer, and providing signs that show the type of entry the respondents should enter (Dillman et al., 2009). An example on a numeric open-ended question could provide a smaller box that fits the maximum number expected and it can provide a label by the box that says the range of numbers expected. The second type of open-ended questions asks respondents to provide a list of items, such as a grocery list (Dillman et al., 2009). The third type of open ended question asked for a descriptive answer and should provide a large space so the respondent feels free to write a complete answer (Dillman et al., 2009).

\section{Research Method}

\section{Data and Sampling}

This study examines the difference in answering open-ended questions between telephone respondents and online respondents. This study utilizes Chi-Square to examine both groups. The first category is the group category which includes telephone respondents and online respondents while the second cate- 
gory is the response or nonresponse for every open-ended item. Open-ended questions include questions about T.V use and consumptions and other questions about their household. Most of the questions are numerical open-ended questions except for one question that asks respondents to provide comments or suggestions.

This study is based on a recent telephone and self-administered survey of PBS members in a local market in a swing state from April 1 to 20,2014. A list of PBS members was used and according to the contact information provided by the members, the researchers divided the members into two groups: telephone and online. Members who provided phone numbers were included in the telephone list and all others were contacted through email.

A simple random sample of members of PBS $(n=275)$ were selected from the PBS database; $(n=53)$ are online respondents and $(n=222)$ are telephone respondents. The study adopted the Tailored Design Method of Dillman (2009) in order to achieve a high response rate. The randomly selected participants were called by trained college students' interviewers and the answers were entered directly to Qualtrics. The non-respondents of the first calls were called again in different times to make sure they are calling in a better time. A total of 275 responses were received from PBS members.

\section{Measurement}

The questionnaire consisted of 44 questions (15 open-ended and 29 closed-ended) on respondents' different levels of T.V use, political participation, and demographic information including gender, age, ethnicity, education, and income level. Only the open-ended questions were chosen for the purposes of the research (see table 1). Chi-square was used to compare online and telephone responses and nonresponses for every open-ended question.

Group (telephone/online). Telephone versus online users were coded as (0) for telephone participants and ( 1 ) for online participants $(M=0.19, S D=0.39)$. Then, every question of the 15 open-ended questions was computed into a different variable and was coded as (1) and nonresponse items were coded as (0).

\section{Results}

To address the research question about whether there is a difference in item-non-response of open ended questions between telephone survey participants and online survey participants, the author conducted a Pearson chi-square. This statistical test is used because both variables are categorical and for the purpose of this study we needed to know the frequencies of the non-response items between both telephone survey participants and online survey participants. The results show statistically significant differences in the non-response items between the two groups (telephone and online surveys). Fifteen open ended questions were statistically analyzed, of which 14 were statistically significant, and the results are as follows: Q11, "How many TVs do you use to watch television?" $\chi 2(2)=8.88, p<.01$. The effect size for this finding, Cramer's $V$ is .18. 90.6\% of the online participants responded to this open ended question compared to $70.7 \%$ of telephone participants. Q12, "How many of them are old analog TVs connected to antenna?" $\chi 2(2)=30.62, p<.001$. The effect size for this finding, Cramer's $V$ is $.33 .90 .6 \%$ of the online participants responded to this open ended question compared to $48.6 \%$ of telephone participants.Q13, "How many of them are old analog TVs connected to cable or satellite?" $\chi 2(2)=34.36, p<.001$. The effect size for this finding, Cramer's $V$ is $.35 .90 .6 \%$ of the online participants responded to this open ended question compared to $45.9 \%$ of telephone participants.Q14, "How many of them are newer digital tuner built in TVs connected to antenna?" $\chi 2(2)=34.7, p<.001$. The effect size for this finding, Cramer's $V$ is $.35 .88 .7 \%$ of the online participants responded to this open ended question compared to $43.7 \%$ of telephone participants. 
Q15, "How many newer digital tuner built in TV connected to cable or satellite?" $\chi 2(2)=21.09, p<.001$. The effect size for this finding, Cramer's $V$ is $.26 .86 .8 \%$ of the online participants responded to this open ended question compared to $52.3 \%$ of telephone participants.Q17, "How many DVRs do you own?" $\chi 2(2)=$ 12.16, $p<.001$. The effect size for this finding, Cramer's V is .21. 88.7\% of the online participants responded to this open ended question compared to $64.0 \%$ of telephone participants.Q18, "How much are you paying for your cable or satellite service? (Just for TV not including internet-- if you don't know, just estimate and write in round up dollars such as 20)." $\chi 2(2)=33.83, p<.001$. The effect size for this finding, Cramer's $V$ is .35. $86.8 \%$ of the online participants responded to this open ended question compared to $42.3 \%$ of telephone participants.Q19, "How many total hours does your household watch TV in a typical week?" $\chi 2(2)=$ 21.52, $p<.001$. The effect size for this finding, Cramer's $V$ is .28. 90.6\% of the online participants responded to this open ended question compared to $56.3 \%$ of telephone participants.Q20, "How much money do you spend for online content subscription services like Netflix or Hulu Plus per month? ( 0 for not using this kind of service: 9999 for don't know what they are)". $\chi 2(2)=28.87, p<.001$. The effect size for this finding, Cramer's $V$ is .32 . $90.6 \%$ of the online participants responded to this open ended question compared to $50.0 \%$ of telephone participants. Q21, "On average, how many hours does your household spend watching paid online programs such as Hulu Plus or Netflix in a typical week?" Pearson Chi-Square is not significant, $\chi^{2}(2)=2.53, p=.074$. The effect size for this finding, Cramer's $V$ is $.96 .24 .5 \%$ of the online participants responded to this open ended question compared to $36 \%$ of telephone participants.Q22, "On average, how many hours does your household spend watching free online programs such as Youtube, Hulu, or TV Network websites in a typical week?" $\chi 2(2)=48.79, p<.001$. The effect size for this finding, Cramer's $V$ is .42 . $86.8 \%$ of the online participants responded to this open ended question compared to $33.8 \%$ of telephone participants.Q35, "How many people do you have in your household?" $\chi 2(2)=25.07, p<.001$. The effect size for this finding, Cramer's $V$ is $.30 .79 .2 \%$ of the online participants responded to this open ended question compared to $41 \%$ of telephone participants. Q36, "How many of them are children?" $\chi 2(2)=$ $36.17, p<.001$. The effect size for this finding, Cramer's $V$ is .36. $79.2 \%$ of the online participants responded to this open ended question compared to $33.8 \%$ of telephone participants.Q37, "How old is/are your child/ children?" $\chi 2(2)=13.52, p<.001$. The effect size for this finding, Cramer's $V$ is $.22 .41 .5 \%$ of the online participants responded to this open ended question compared to $18.0 \%$ of telephone participants.Q44, "Do you have any other comments or questions for WBGU-TV?" $\chi 2(2)=15.18, p<.001$. The effect size for this finding, Cramer's Vis .23.37.7\% of the online participants responded to this open ended question compared to $14.4 \%$ of telephone participants (see table 1 ).

\section{Discussion}

This paper shows the difference between online surveys and telephone surveys in using open ended questions. Previous studies have investigated the differences between different survey modes and the use of mixed-mode methodologies. However, few studies have discussed open ended questions. The development of information and communication technologies has encouraged many researchers to use online surveys alone or with another mode in order to complement and reduce errors. Dillman et al. (2009) indicate that the design of a question can be measured differently from one mode to another and they gave an example of how a question was answered as an open ended question over the phone while answered as a closed ended question online. Therefore, this study examines a set of fifteen open ended questions that were asked to two samples online and over the telephone. The results of this study show significant differences between the two groups in answering the open ended items. Online respondents were significantly higher than telephone respondents in answering open ended questions. This significant difference was shown in fourteen of the fifteen questions analyzed. One question did not have any significant difference between the two groups which was "On average, how many hours does your household spend watching paid online 
programs such as Hulu Plus or Netflix in a typical week?" In this item telephone respondents answered a few more questions than online however, in all the other fourteen questions, online respondents answered more than the telephone respondents. The findings of our study are consistent with previous studies that indicate that individuals tend to feel more at ease when answering questions when they are not placed on the spot by an interviewer but rather, can take the surveys privately. In addition, they may be able to re-read the questions if they do not understand them completely the first time they read it versus telephone interviews where respondents sometimes feel uncomfortable during pauses of silence where they do not have an immediate answer. They may also be more reluctant to answer open ended questions when an interviewer is conducting the survey out of fear of being judged (Chang \& Krosnick, 2009).

A limitation of this study is that the telephone sample $(n=222)$ is larger than the online sample $(n=45)$. Future studies should aim to increase the sample size of online participants and see if there are different findings. Another limitation is that this sample was drawn from a special population in a community which supports a local television station in Northwest Ohio. The survey questions asked about that local television station, which they support. Future studies could investigate different samples that are representative of a larger population. Furthermore, this study examined one type of survey question, which is open ended questions). Future studies could investigate this research question from different perspectives such as comparing closed ended questions with open ended questions. Lastly, future studies could include more variables, such as demographics and socio-economic status variables to see if these variables have an effect on the response rate besides the survey mode itself.

\section{Conclusion}

The emergence of the internet has introduced a new mode of survey (online surveys). Many studies have rapidly begun to use online surveys as a method. However, online surveys have disadvantages such as their difficulty in allowing researchers to collect truly random samples. The method for collecting contact information of respondents is not as simple as random digit dialing in telephone surveys. Due to email address varieties, it is difficult to send surveys to random people. In addition, the use of the internet varies more so than the use of telephone in terms of who can navigate the web and who has access to it (Dillman et al., 2009). Therefore, researchers supplement these online surveys with a second survey mode, thus balancing these disadvantages. Certain questions however, can be answered differently depending on which mode is used to pose the question, which raises reliability issues.

This study investigates a particular type of survey question (open-ended) and how they are answered depending on the survey mode (telephone or online). The findings show a significant difference between online and telephone respondents in answering open ended questions. Online participants are more likely to respond to open ended questions than telephone participants. This result supports other previous studies that argue that online participants are more comfortable answering certain questions when there is no interviewer facilitating the survey. Participants tend to feel more anonymous when answering certain questions at their own pace and in private (Chang \& Krosnik, 2009). 


\section{References}

- $\quad$ Biemer, P. P. \& Lyberg, L. E. (2003). Introduction to Survey Quality, Vol. 335. John Wiley \& Sons.

- $\quad$ Chang, L. \& Krosnick, J. A. (2009). “National Surveys Via RDD Telephone Interviewing Versus the Internet Comparing Sample Representativeness and Response Quality", Public Opinion Quarterly, 73 (4), 641-678.

- Czaja, R. \& Blair, J. (1996). Designing Surveys: A Gguide to Decision and Evaluation. London.

- Dillman, D. A.; Eltinge, J. L.; Groves, R. M. \& Little, R. J. (2002). "Survey Nonresponse in Design, Data Collection, and Analysis", Survey Nonresponse, 3-26.

- Dillman, D. A.; Smyth, J. D. \& Christian, L. M. (2009). Internet, Mail, and Mixed-mode Surveys: The Tailored Design Method. Hoboken.

- Fowler, F. J.; Roman, A. M. \& Di, Z. X. (1998). “Mode Effects in a Survey of Medicare Prostate Surgery Patients", Public Opinion Quarterly, 62 (1), 29.

- Groves, R. M. (2004). Survey Errors and Survey Costs, Vol. 536. John Wiley \& Sons.

- Hoonakker, P. \& Carayon, P. (2009). “Questionnaire Survey Nonresponse: A Comparison of Postal Mail and Internet Surveys", Intl. Journal of Human-Computer Interaction, 25(5), 348-373.

- Hox, J. J. \& De Leeuw, E. D. (1994). A comparison of nonresponse in mail, telephone, and face-toFace surveys", Quality and Quantity, 28 (4), 329-344.

- $\quad$ Schaefer, D. R. \& Dillman, D. A. (1998). “Development of a Standard e-mail Methodology: Results of an Experiment", Public Opinion Quarterly, 378-397. 


\section{Appendix}

Table 1: Chi-square showing the difference in item-response between telephone and online survey

\begin{tabular}{|c|c|c|c|}
\hline Questions & Telephone & Online & $\chi 2$ \\
\hline Q11: How many TV's do you use to watch TV? & 70.7 & $90.60 \%$ & $8.88^{* *}$ \\
\hline Q12: How many of them are old analog TVs connected to antenna? & $48.60 \%$ & $90.60 \%$ & $30.62^{* * *}$ \\
\hline Q13: How many of them are old analog TVs connected to cable or satellite? & $45.90 \%$ & $90.60 \%$ & $34.36^{* * *}$ \\
\hline Q14: How many of them are newer digital tuner built in TV connected to antenna? & $43.70 \%$ & $88.70 \%$ & $34.7^{* * *}$ \\
\hline Q15: How many newer digital tuner built in TV connected to cable or satellite? & $52.30 \%$ & $86.80 \%$ & $21.09 * * *$ \\
\hline Q17: How many DVRs do you own? & $64 \%$ & $88.70 \%$ & $12.16^{* * *}$ \\
\hline Q18: How much are you paying for your cable or satellite service? & $42.30 \%$ & $86.80 \%$ & $33.83^{* * *}$ \\
\hline Q19: How many total hours does your household watch TV in a typical week? & $56.30 \%$ & $90.60 \%$ & $21.52^{* * *}$ \\
\hline $\begin{array}{l}\text { Q20: How much money do you spend for online content subscription services like Netflix or } \\
\text { Hulu Plus per month? }\end{array}$ & $50 \%$ & $90.60 \%$ & $28.87^{* * *}$ \\
\hline $\begin{array}{l}\text { Q21: On average, how many hours does your household spend watching paid online Pro- } \\
\text { grams such as Hulu Plus or TV Netflix in a typical week? }\end{array}$ & $36 \%$ & $24.50 \%$ & 2.53 \\
\hline $\begin{array}{l}\text { Q22: On average, how many hours does your household spend watching free online Pro- } \\
\text { grams such as Youtube, Hulu, or TV Network websites in a typical week? }\end{array}$ & $33.80 \%$ & $86.80 \%$ & $48.79 * * *$ \\
\hline Q35: How many people do you have in your household? & $41 \%$ & $79.20 \%$ & $25.07 * * *$ \\
\hline Q36: How many of them are children? & $33.80 \%$ & $79.20 \%$ & $36.17^{* * *}$ \\
\hline Q37: How old is/are your child/children? & $18 \%$ & $41.50 \%$ & $13.52^{* * *}$ \\
\hline Q44: Do you have any other comments or questions for WBGU-TV? & $14.40 \%$ & $37.70 \%$ & $15.18^{* * *}$ \\
\hline
\end{tabular}

Note: $\mathrm{N}=275 * \mathrm{p}<.05 .{ }^{*} \mathrm{p}<.01 . * * * \mathrm{p}<.001 .$. 